\title{
Structured and Unstructured Robust Control for an Induction Motor
}

\author{
Jhoel F. Espinoza-Quispe ${ }^{1}$, Juan C. Cutipa-Luque ${ }^{2}$, German A. Echaiz Espinoza ${ }^{3}$, Andres O. Salazar ${ }^{4}$ \\ Electronic Engineering Professional School \\ Universidad Nacional de San Agustín de Arequipa, Arequipa, Peru ${ }^{1,2,3}$ \\ Computer and Automation Engineering Department \\ Universidade Federal de Rio Grande do Norte, Natal, Brazil ${ }^{4}$
}

\begin{abstract}
The indirect field-oriented control approaches for induction motors have recently gained more attention due to its use in trend areas, such as electromobility, electric vehicles, electric ships, and unmanned vehicles. This work studies the performance of two advanced control synthesized by the $\mathcal{H}_{\infty}$ norm as an alternative to the classical Proportional-Integral and Derivative controller. It will be assessed in terms of the performance against disturbance variations in the reference speed in the nominal conditions. The tuning of the parameters of these controllers must be defined of the stability and performance of the system and to increase their operation range frequency. An algorithm is proposed to reach a better shape of weighting functions. A numerical simulation will be shown where the advances in structured advanced controller synthesis with unstructured $\mathcal{H}_{\infty}$ controller is still the good election for the induction motor control. Unstructured controller approach shows still good robustness in performance and stability compared with the structured controller. Constraints imposed in structured controller is the main disadvantage to improve its robustness properties. However, compared with a conventional PID approach, the structured controller has shown quite good performance and can become in one of the most attractive approaches for practitioners.
\end{abstract}

Keywords- $\mathcal{H}_{\infty}$ Robust control; induction motor; indirect field oriented control

\section{INTRODUCTION}

Nowadays, advances in research and technology seek to tackle the problem of air pollution. The study of control approaches for induction motor has gained attention due its massive use in electric vehicles and other solution involving electromobility. There are two main strategies used in induction motor control, the first is the direct torque control (DTC) and the second is the indirect field-oriented control (IFOC) [1]. The trend in innovation is the use of advanced controllers, the latest published works highlight that the IFOC is a high performance system, but it does not guarantee robustness in performance and in stability. In [1], the authors evaluate the performance between conventional Proportional-Integrative and advanced controllers with DTC and IFOC strategies. The main disadvantage in PI controller is the presence of high overshoot peak that may be reduced with modified PI structures, such as used in [2]. The advanced controllers presented quite good performance in energy efficiency relative to fuzzy logic and conventional PI controllers. The sliding mode control approach presented good speed tracking and energy efficiency, but the chattering effect may cause high frequency vibration and damage the electromechanical pieces, such as bearings and transmission gears in the powertrain [3], [4].
The unstructured $\mathcal{H}_{\infty}$ robust control applied to an induction motor is presented in [5] and further works considered gain scheduling and current controller [6], [7]. Recent approaches seeks to improve the performance of structured controller using the linear matrix inequalities for optimization [8], [9]. These approaches present a better tuning procedures to achieve the robustness in performance and stability and guaranteed the user requirements. In [10], the authors present emerging concept related to control of mechatronic systems, putting the loop shaping design as a good tool for synthesis of industrial controllers and remarking the $\mathcal{H}_{\infty}$ as a good alternative in advanced control due to the robustness. In [11], the authors present a $\mathcal{H}_{\infty}$ controller synthesis including a model reference adaptive estimator to avoid the use of encoders. Other authors propose a robust control approach applied to ship propulsion electric motor [12]. Another advanced control approaches [13], [14], [15], [16] are also applied to induction motors, but this work is regarding the comparison of the two types which have in common the $\mathcal{H}_{\infty}$ norm for the controller synthesis.

The organization of this paper follows: Section I presents the introduction over the induction motor control approaches, Section II presents the mathematical model of the induction motor, Section III presents the robust control approaches (structured and unstructured), Section IV presents the numerical results and Section $\mathrm{V}$ provides the conclusions.

\section{MOTOR MODEL}

Using the park transform, there are three general models to represent an induction motor. The first is based on an arbitrary rotating reference frame, the second is based on a synchronous rotating reference frame, and the third is based on a stationary reference frame. In this work, following the nomenclature in [17], the $q d 0$ stationary reference frame is used to model the induction motor where the equations relative to the stator are:

$$
\begin{aligned}
& v_{q s}^{s}=\frac{N}{\omega_{b}} \psi_{q s}^{s}+r_{s} i_{q s}^{s} \\
& v_{d s}^{s}=\frac{N}{\omega_{b}} \psi_{d s}^{s}+r_{s} i_{d s}^{s} \\
& v_{0 s}=\frac{N}{\omega_{b}} \psi_{0 s}+r_{s} i_{0 s}
\end{aligned}
$$

where $v_{q s}^{s}, v_{d s}^{s}$, and $v_{0 s}$ are the stator voltages; $\psi_{q s}^{s}, \psi_{d s}^{s}$, and $\psi_{0 s}$ are their magnetic flux; $i_{q s}^{s}, i_{d s}^{s}$, and $i_{0 s}$ are their electric currents; $N$ is the number of poles; $\omega_{b}$ is the base electrical 
frequency; and $r_{s}$ is the stator resistance.

Similarly, the equations relative to the rotor are:

$$
\begin{gathered}
v_{q r}^{\prime s}=\frac{N}{\omega_{b}} \psi_{q r}^{\prime s}-\frac{\omega_{r}}{\omega_{b}} \psi_{d r}^{\prime s}+r_{r}^{\prime} i_{q r}^{\prime s} \\
v_{d r}^{\prime s}=\frac{N}{\omega_{b}} \psi_{d r}^{\prime s}+\frac{\omega_{r}}{\omega_{b}} \psi_{q r}^{\prime s}+r_{r}^{\prime} i_{d r}^{\prime s} \\
v_{0 r}^{\prime}=\frac{N}{\omega_{b}} \psi_{0 r}^{\prime}+r_{r}^{\prime} i_{0 r}^{\prime}
\end{gathered}
$$

where $v_{q r}^{\prime s}, v_{d r}^{\prime s}$, and $v_{0 r}^{\prime}$ are the rotor voltages; $\psi_{q r}^{\prime s}, \psi_{d r}^{\prime s}$, and $\psi_{0 s}^{\prime}$ are their magnetic flux; $i_{q r}^{\prime s}, i_{d r}^{\prime s}$, and $i_{0 s}^{\prime}$ are their electric currents; $N$ is the number of poles; $\omega_{b}$ is the base electrical frequency; $r_{r}^{\prime}$ is the rotor resistance; and $\omega_{r}$ is the rotor angular frequency.

Considering $\Psi=\left[\psi_{q s}^{s}, \psi_{d s}^{s}, \psi_{0 s}, \psi_{q r}^{\prime s}, \psi_{d r}^{\prime s}, \psi_{0 r}^{\prime}\right]^{T}$ as the magnetic flux vector and $I=\left[i_{q s}^{s}, i_{d s}^{s}, i_{0 s}, i_{q r}^{\prime s}, i_{d r}^{\prime s}, i_{0 r}^{\prime}\right]^{T}$ as the current vector, the following relation can be expressed:

$$
\Psi=X_{s r} . I,
$$

where $X_{s r}$ is the stator-rotor reactance matrix equivalent to:

$$
X_{s r}=\left[\begin{array}{ll}
X_{11} & X_{12} \\
X_{21} & X_{22}
\end{array}\right]
$$

with:

$$
\begin{gathered}
X_{11}=\left[\begin{array}{ccc}
x_{l s}+x_{m} & 0 & 0 \\
0 & x_{l s}+x_{m} & 0 \\
0 & 0 & x_{l s}
\end{array}\right], \\
X_{22}=\left[\begin{array}{ccc}
x_{l r}^{\prime}+x_{m} & 0 & 0 \\
0 & x_{l r}^{\prime}+x_{m} & 0 \\
0 & 0 & x_{l r}^{\prime}
\end{array}\right], \\
X_{12}=X_{21}=\left[\begin{array}{ccc}
x_{m} & 0 & 0 \\
0 & x_{m} & 0 \\
0 & 0 & 0
\end{array}\right],
\end{gathered}
$$

where $x_{l s}$ is the stator leakage reactance, $x_{m}$ is the stator magnetizing reactance, and $x_{l r}^{\prime}$ is the rotor leakage reactance. All parameter value can be found in Table I.

The motor electromagnetic torque $T_{e m}$ is defined as:

$$
T_{e m}=\frac{3}{2} \frac{N}{2 \omega_{b}}\left(\psi_{d s}^{s} \cdot i_{q s}^{s}-\psi_{q s}^{s} \cdot i_{d s}^{s}\right),
$$

and the 'per unit of speed relation' with the externally-applied mechanical torque $T_{\text {mech }}$ (in the same direction of rotor speed) and the damping torque $T_{d a m p}$ (in the opposite direction of motor speed) is expressed as follows:

$$
T_{e m}+T_{m e c h}-T_{d a m p}=2 H \frac{d}{d t}\left(\frac{\omega_{r}}{\omega_{b}}\right),
$$

where $H=J \omega_{b m}^{2} / 2 S_{b}$ is the inertia constant, $J$ is the rotor inertia, $\omega_{b m}$ is the base mechanical frequency, and $S_{b}$ is rated volt-ampere. Table I shows the main parameter values of the 20 HP induction motor used in this work for numerical validation.

The whole above relations and differential equations should be linearized for their use in synthesis of proposed control approaches. In this work, the nonlinear model is represented in Simulink to be used numerical approximation for linearization
TABLE I. PARAMETER VALUES OF THE INDUCTION MOTOR

\begin{tabular}{|c|c|c|c|}
\hline Parameters & Symbols & Values & Units \\
\hline \hline Stator winding resistance & $r_{s}$ & 0.1062 & $\Omega$ \\
\hline Stator leakage reactance & $x_{l s}$ & 0.2145 & $\Omega$ \\
\hline Stator magnetizing reactance & $x_{m}$ & 5.8339 & $\Omega$ \\
\hline Rotor leakage reactance & $x_{l r}^{\prime}$ & 0.2145 & $\Omega$ \\
\hline Rotor winding resistance & $r_{r}^{\prime}$ & 0.0764 & $\Omega$ \\
\hline Number of poles & $N$ & 4 & - \\
\hline Moment of inertia & $J$ & 2.8 & $\mathrm{Kg.m}$ \\
\hline Rated voltage & $V_{\text {rated }}$ & 220 & $v$ \\
\hline Rated frequency & $f_{\text {rated }}$ & 60 & $\mathrm{~Hz}$ \\
\hline Rated speed & $N_{\text {rated }}$ & 1748.3 & $\mathrm{rpm}$ \\
\hline
\end{tabular}

through the Matlab 'linmod' command in order to obtain the following linear time invariant representation:

$$
\begin{aligned}
& \dot{x}=A x+B u, \\
& y=C x+D u,
\end{aligned}
$$

where $x$ is the state vector, $u$ is the control vector, $y$ is the measurement, $A$ is the state matrix, $B$ is the control matrix, $C$ is the output matrix and $D$ is the feedthrough matrix. The relation between output and input in the $s$ Laplace domain results in the transfer function of the system:

$$
G(s)=\frac{y(s)}{u(s)}=C(s I-A)^{-1} B+D
$$

Table II presents the poles and zeros of three reducedorder models of the system $G(s)$, commonly found in the literature. The reduced-order models have been be obtained using Hankel singular values that indicate the respective state energy of the system. The 5th reduced-order model is used for controller syntheses and the 2 nd reduced-order model is used to find the natural frequency $\omega_{0}=0.21 \mathrm{rad} / \mathrm{s}$ to shape properly the sensitivity functions to improve the performance and robustness of the $\mathcal{H}_{\infty}$ controllers.

TABLE II. Poles AND ZERos of THE REDUCED-ORder Models For $G(s)$

\begin{tabular}{|c|c|c|c|}
\hline & 9th order & 5th order & 2nd order \\
\hline Poles & Value & Value & Value \\
\hline p1 & 0 & 0 & 0.2121 \\
\hline p2 & -2850766.0028 & $-4.7652+3.7675 j$ & -0.2043 \\
\hline p3 & -1451235.2842 & $-4.7652-3.7675 j$ & \\
\hline p4 & -1451235.2313 & 0.2121 & \\
\hline p5 & -113.0944 & -0.2052 & \\
\hline p6 & $-4.7652+3.7675 j$ & & \\
\hline p7 & $-4.7652-3.7675 j$ & & \\
\hline p8 & 0.2121 & & \\
\hline p9 & -0.2052 & & Value \\
\hline \hline Zeros & Value & Value & \\
\hline z1 & 0 & 0 & \\
\hline z2 & -2850766.0028 & -4.7619 & \\
\hline z3 & -1451235.2579 & $-3.5928+2.0923 j$ & \\
\hline z4 & -113.0944 & $-3.5928-2.0923 j$ & \\
\hline z5 & -4.7619 & & \\
\hline z6 & $-3.5928+2.0923 j$ & & \\
\hline z7 & $-3.5928-2.0923 j$ & & \\
\hline
\end{tabular}

\section{CONTROL APPROACH}

This section presents two control approaches based in loop shaping design, or well known as the $\mathcal{H}_{\infty}$ mixed sensitivity approach. The first approach leads to get an $\mathcal{H}_{\infty}$ controller 
with no defined structure, named unstructured controller. The second approach leads to get an $\mathcal{H}_{\infty}$ controller with a conventional PID structure, named structured controller. Both approaches use sensitivity weighting functions for loop shaping in order to get a robustness in performance and stability [18], [19].

\section{A. Unstructured Controller}

Fig. 1 shows $G$, the 5 th order induction motor system represented by (15), inserted into the augmented plant $P$ with two port representation commonly used in the $\mathcal{H}_{\infty}$ controller synthesis. The diagram also contains the weighting functions $W_{*}$, the exogenous output $z$, the exogenous input $w$, the control variable $u$ and the controlled variable $y$. According to this representation, $w$ is equivalent to the reference rotor speed reference signal $\omega_{r}(r e f), u$ is equivalent to the electromagnetic torque signal $T_{e m}^{*}, y$ is the measured rotor speed signal $\omega_{r}$. And $z$ contains the error between reference and measured rotor speed, the electromagnetic torque control, and the measured rotor speed, weighting respectively by functions $W_{P}, W_{u}$, and $W_{T}$.

The problem of unstructured control is defined here as to find a rotor speed controller $K$ that minimizes the $\mathcal{H}_{\infty}$ norm of the transfer function between exogenous output $z$ and exogenous input $w$ as follows [18]:

$$
\left\|T_{z w}\right\|=\left\|W_{P} S W_{u} K S W_{T} T\right\|_{\infty}^{T}
$$

where the sensitivity function $S$, the complementary sensitivity function $T$ and the control sensitivity function $K S$ are defined respectively as:

$$
\begin{gathered}
S=(I-G K)^{-1} \\
T=(I-G K)^{-1} G K \\
K S=(I-G K)^{-1} K
\end{gathered}
$$

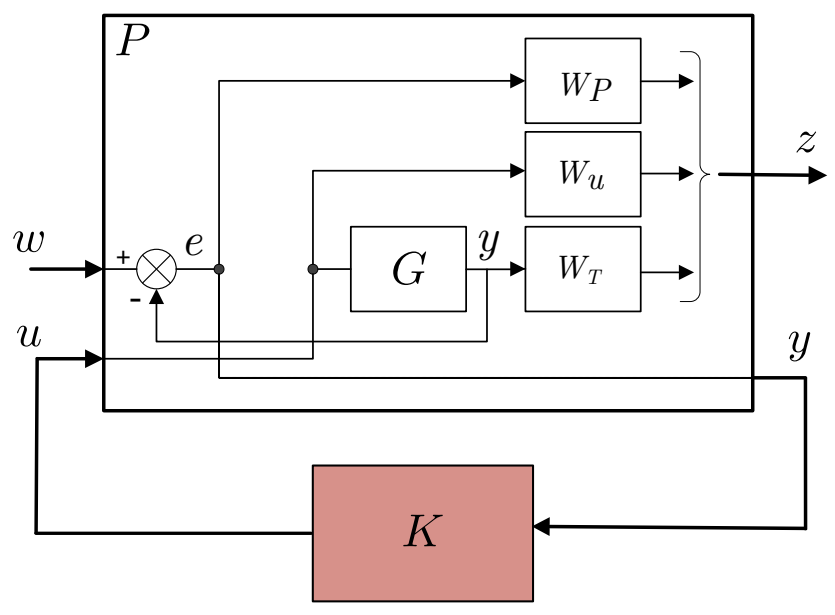

Fig. 1. Unstructured $\mathcal{H}_{\infty}$ Controller.

\section{B. Structured Controller}

Fig. 2 shows $G$, the 5 th order induction motor system represented by (15), inserted also into the augmented plant $P$ with two port representation. The diagram contains the weighting functions $W_{*}$, the exogenous output $z$, the exogenous input $w$, the control variable $u$ and the controlled variable $y$. And contains all variables at same representation in the last section with the only difference that the controller $K$ has now a defined PID structure:

$$
K=k_{p}+k_{i} \frac{1}{s}+k_{d} s,
$$

The problem of structured control is defined here as to find a rotor speed controller $K$ that minimizes the $\mathcal{H}_{\infty}$ norm of the transfer function between exogenous output $z$ and exogenous input $w$ as follows [19]:

$$
\left\|T_{z w}\right\|=\left\|W_{P} S W_{T} T\right\|_{\infty}^{T}
$$

subject to structure constraints given in (20). It is important to note the electromagnetic torque control signal is not included in the above matrix to avoid degradation in performance due to limited constraints given to $K$ structure.

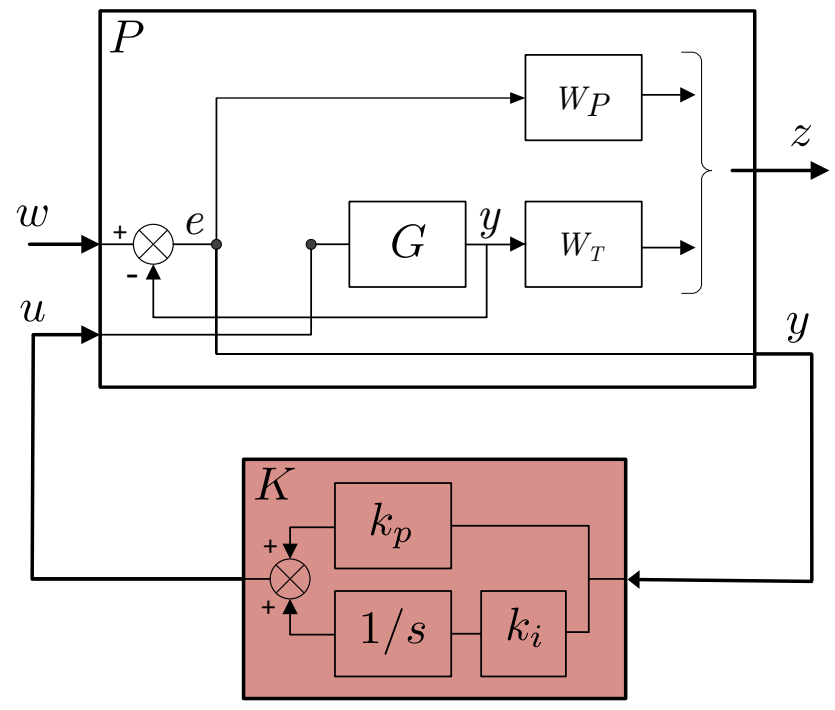

Fig. 2. Structured $\mathcal{H}_{\infty}$ Controller.

\section{Computation Resources}

The problem of minimization of $\mathcal{H}_{\infty}$ norm of equations (16) and (21) will be solved using computational resources available in commercial and non-commercial software, such as Matlab, Gnu-Octave, and Scilab. This solution is based in the algebraic Riccati equation, or alternatively putting the problem in the linear and bi-linear matrix inequalities for respective optimization [20]. Assuming that the involved matrices in 15 satisfy detectability and stabilizability conditions, there is a suboptimal controller $K$ that the closed function $T_{z w}$ achieves:

$$
\left\|T_{z w}\right\|=\gamma,
$$

where $\gamma$ is a real number related with the suboptimal control problem. The design requirements for control of an induction motor is given in terms of closed loop sensitivities: 
1) Closed loop stability,

2) $\bar{\sigma}(S)<1$ for $\omega<\omega_{P}$,

3) $\bar{\sigma}(T)<1$ for $\omega>\omega_{T}$,

4) $\bar{\sigma}(K S)<1$ for $\omega>\omega_{u}$,

where the crossover frequencies of the sensitivity functions are $\omega_{P}, \omega_{T}$ and $\omega_{u}$, respectively. These frequencies are commonly set according to good tracking, good disturbance and noise rejection, and model uncertainties. In this work, the goal is to improve still more these requirements and these crossover specification frequencies are also tuned using a frequency sweep algorithm as shown below.

\section{Frequency Sweep}

The design requirements given through sensitivity functions $S, T$ and $K S$ are weighting with $W_{P}, W_{T}$ and $W_{u}$ according to:

$$
\begin{aligned}
W_{P}(s) & =\frac{s / M_{P}+\omega_{P}}{s+\omega_{P} A_{P}} \\
W_{T}(s) & =\frac{s+\omega_{T} A_{T}}{s / M_{T}+\omega_{T}} \\
W_{u}(s) & =\frac{s+\omega_{u} A_{u}}{s / M_{u}+\omega_{u}}
\end{aligned}
$$

where $M_{P}, M_{T}, M_{u}, A_{P}, A_{T}$ and $A_{u}$ are constants. In this work, both the unstructured and structured control synthesis use a sweeping in frequency in its cutoff frequencies in order to improve the operation bandwidth.

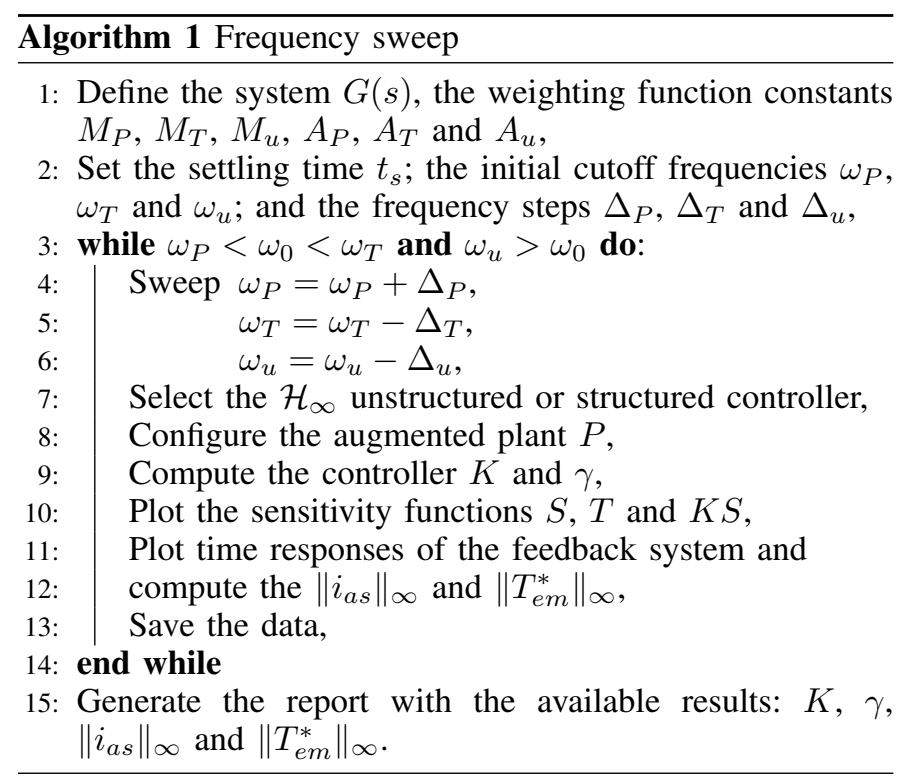

The sweeping in frequency starts with initial cutoff frequencies given around the natural frequency $\omega_{0}$, the final report identifies the absolute maximum values of the stator current $i_{a s}$ and the electromagnetic torque $T_{e m}^{*}$. This algorithm enables to observe the water bed effect between performance and stability in robust control approaches, aids to select a better controller $K$ between a set, ensures the control variable do not exceed physical limits, and guarantees good performance according to the settling time $t_{s}$ and the zero steady state error.

\section{RESULTS}

This section presents the results of the two control approaches used in this work. Fig. 3 presents the simulator of an induction motor on Simulink, where the $q d 0$ transform is explicit, the block of robust speed controller contains the controller (unstructured or structured), the speed rotor reference signal is denoted by $\omega_{r}(r e f)$, and other related components of the dynamic model [17].

\section{A. Unstructured Robust Control}

The initial cutoff frequencies were set to $\omega_{P}=0.01 \mathrm{rad} / \mathrm{s}$, $\omega_{T}=1 \mathrm{rad} / \mathrm{s}, \omega_{u}=2000 \mathrm{rad} / \mathrm{s}$. After a number of 100 iterations running into the sweep algorithm, the report shows a better achieved gamma value of $\gamma=0.9549$ with cutoff frequencies of $\omega_{P}=0.01 \mathrm{rad} / \mathrm{s}, \omega_{T}=0.9 \mathrm{rad} / \mathrm{s}, \omega_{u}=1000$ $\mathrm{rad} / \mathrm{s}$. The obtained suboptimal unstructured controller $K$ is of 7 th order. Fig. 4 presents the sensitivities $(S, T, K S)$ and their respective weightings $\left(W_{P}, W_{T}, W_{u}\right)$ in the frequency domain response, there is no crossing between each sensitivity and its respective weighting that confirms the good robustness in performance and stability.

\section{B. Structured Robust Control}

The initial cutoff frequencies were set to $\omega_{P}=0.01 \mathrm{rad} / \mathrm{s}$, $\omega_{T}=1 \mathrm{rad} / \mathrm{s}$. After a number of 100 iterations running into the sweep algorithm, the report shows an achieved gamma value of $\gamma=23.7674$ with cutoff frequencies of $\omega_{P}=0.01$ $\mathrm{rad} / \mathrm{s}, \omega_{T}=0.1 \mathrm{rad} / \mathrm{s}$. The obtained suboptimal unstructured controller $K$ has a PID structure (20) with $k_{p}=163.3002$, $k_{i}=0.0016$ and $k_{d}=0$. Fig. 5 presents the sensitivities $(S$, $T)$ and their respective weightings $\left(W_{P}, W_{T}\right)$ in the frequency domain response. Despite the good loop shaping in $S$ and $T$, there are crossings between each sensitivity and its respective weighting that confirms the poor robustness in performance and stability. This means that the unstructured controller has better properties of disturbance rejections, and the constraints given in this structured controller have limited the suboptimal solution to high gamma values.

\section{Time Domain Responses}

This section presents numerical results using a simulator when the system is subjected to the reference rotor speed $\omega_{r}(r e f)$ and the required mechanical torque $T_{m e c h}$. The $\omega_{r}(r e f)$ starts in zero and increases in ramp mode to reach a nominal value of the motor $188.5 \mathrm{rad} / \mathrm{s}$. The required mechanical torque is a pulsating signal given in function of the machine nominal torque $81.49 \mathrm{Nm}$ as shown in Fig. 6.

Fig. 7 presents the time domain responses of $v_{a g}$, one of the three-phase voltages applied to the stator. Considering the structured controller (top side), the $\left\|v_{a g}\right\|_{\infty}$ appears in the first mechanical torque variation, reaching $552.0 \mathrm{~V}$. Considering the unstructured controller (bottom side), the $\left\|v_{a g}\right\|_{\infty}$ appears in the first mechanical torque variation, reaching $286.1 \mathrm{~V}$. It is observed high step variation in mechanical torque produces a peak in the voltage to compensate this demand.

Fig. 8 presents the time domain responses of $i_{a s}$, one of the three-phase current applied to the stator. Considering the structured controller (top side), the $\left\|i_{a s}\right\|_{\infty}$ appears in the 


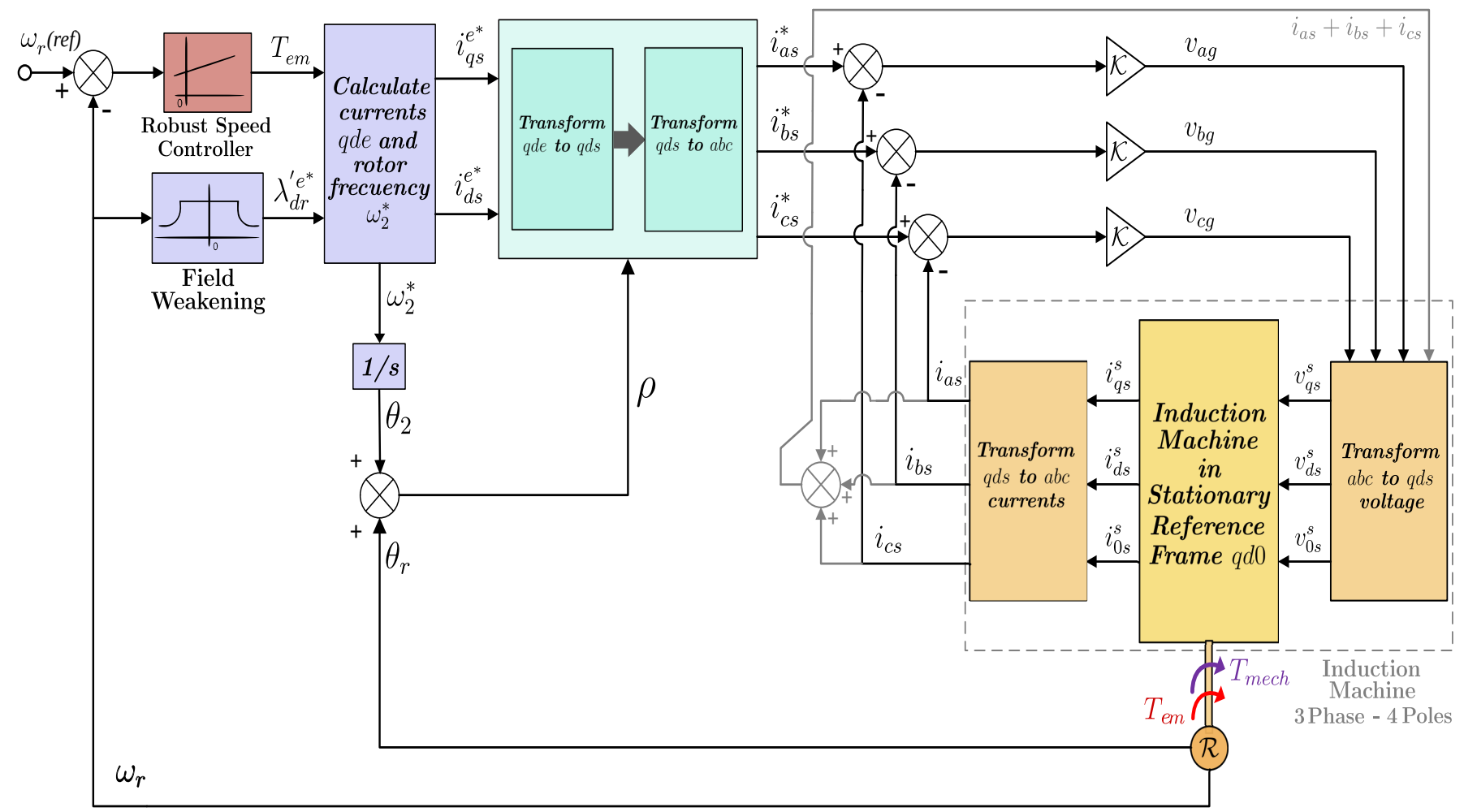

Fig. 3. Simulator of Induction Motor in Simulink.

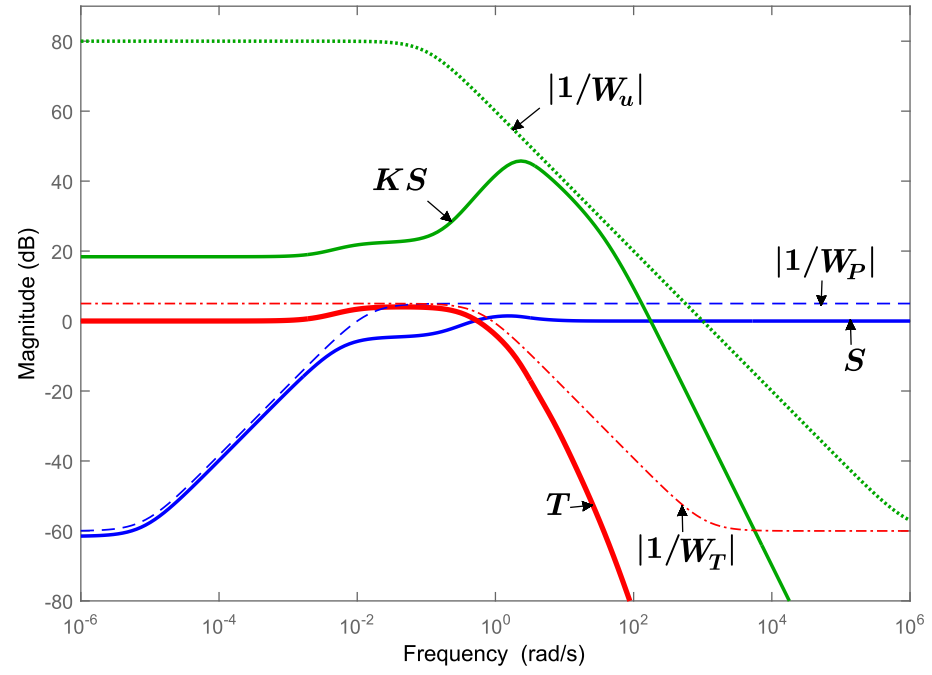

Fig. 4. Sensitivities Unstructured $\mathcal{H}_{\infty}$ Controller.

starting motor, reaching 300.4 A. Considering the unstructured controller (medium side), the $\left\|i_{a s}\right\|_{\infty}$ appears in the starting motor, reaching 211.0 A. Considering the conventional PI controller, tuned as in [17], (bottom side), the $\left\|i_{a s}\right\|_{\infty}$ appears in the starting motor, reaching $147.4 \mathrm{~A}$. It is observed high mechanical torque produces increments in the currents to compensate this demand.

Fig. 9 presents a comparison between controlled variables, speed rotor $\omega_{r}$, using unstructured, structured and PI controllers. At right side, zoom shows the structured controller

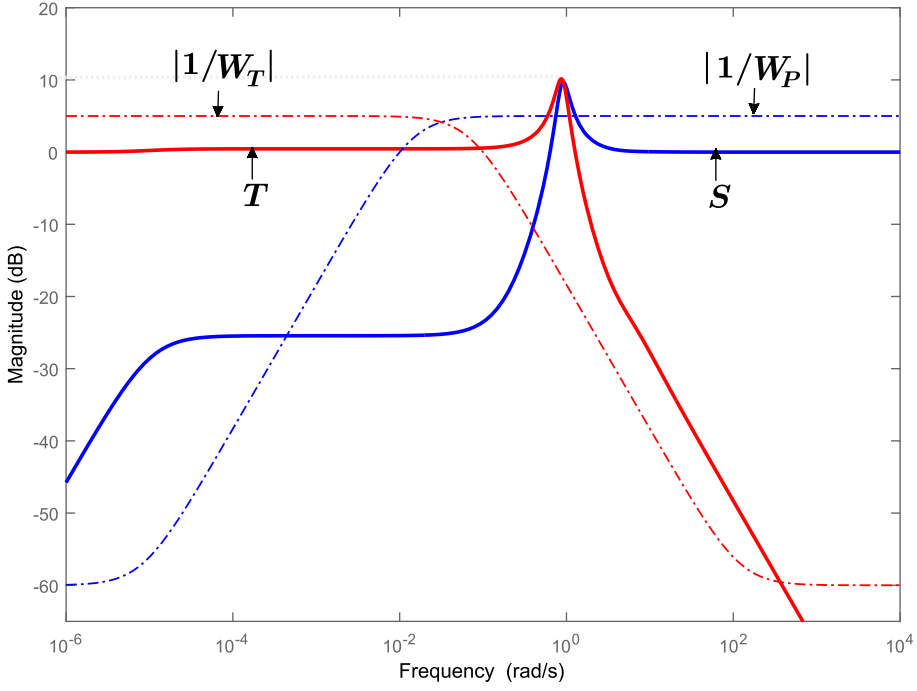

Fig. 5. Sensitivities Structured $\mathcal{H}_{\infty}$ Controller.

has the best tracking despite the variation in mechanical torque, following by the unstructured controller. The poor performance in these results is getting by the conventional PI controller, included the significant error in steady state.

Fig. 10 presents a comparison of control variable responses, electromagnetic torque $T_{e m}$, using unstructured, structured and PI conventional controllers. The structured controller has the maximum $\left\|T_{e m}\right\|_{\infty}=288 \mathrm{Nm}$ between the three controllers. The lowest $\left\|T_{e m}\right\|_{\infty}=141 \mathrm{Nm}$ is related with the con- 


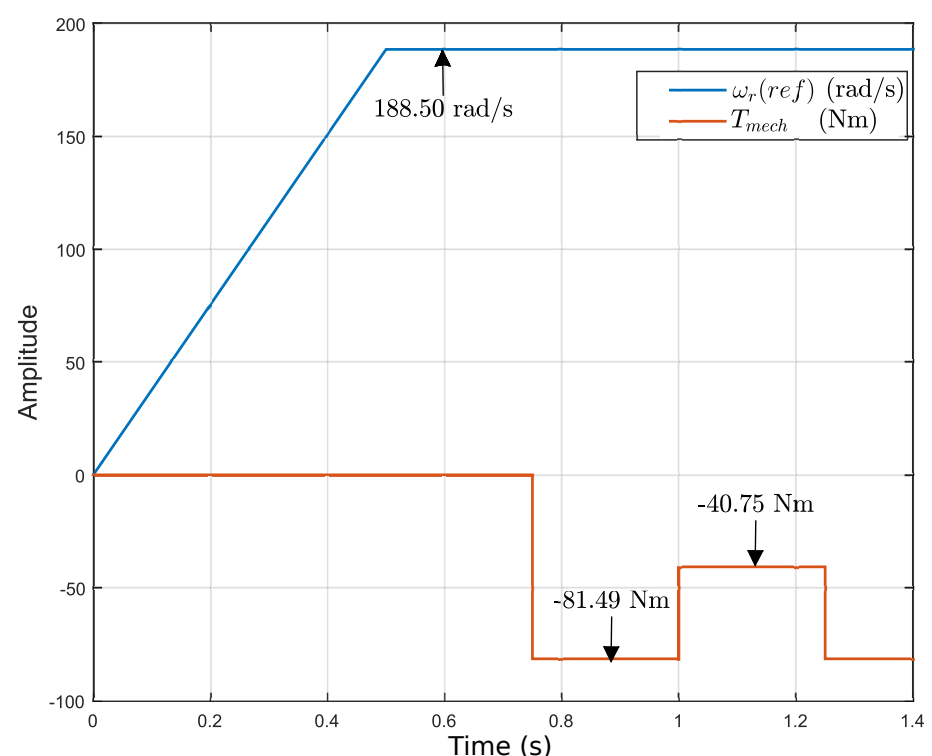

Fig. 6. Reference Speed $\omega_{r}(r e f)$ and Mechanical Torque $T_{m e c h}$.
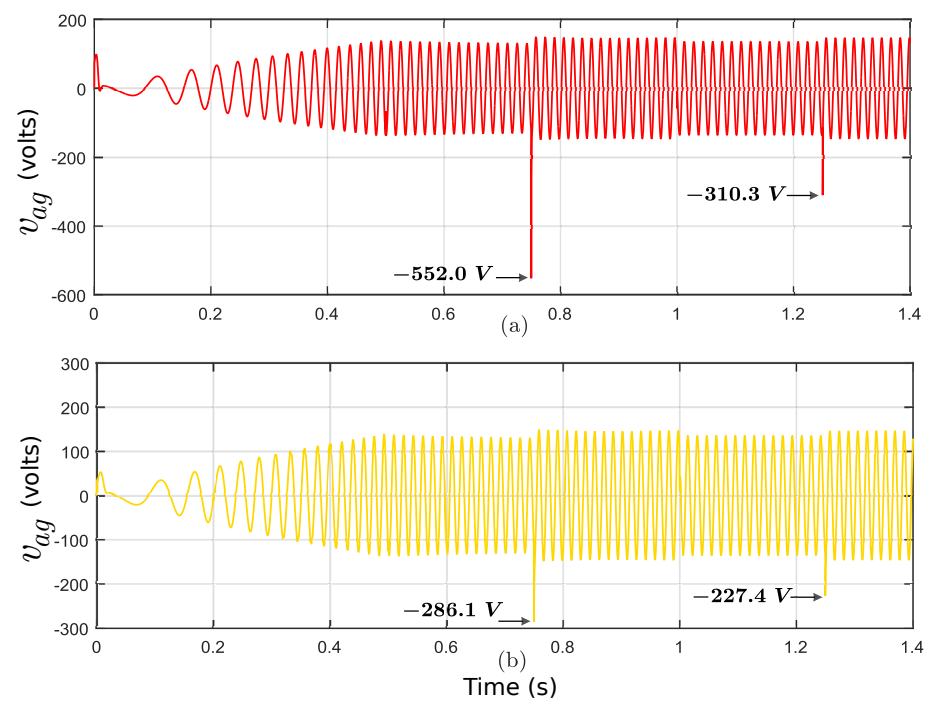

Fig. 7. The Stator Voltage $v_{a g}$ Responses with $\mathcal{H}_{\infty}$ Controllers: (a) Structured Controller, (b) Unstructured Controller.

ventional PI controller. The moderate results are getting by the unstructured controller. However, there are no significant difference in the steady state.

Despite the use of $\mathcal{H}_{\infty}$ approach to synthesis structured controller [18], [7], there is no information regarding the synthesis of structured controller using the same norm. Relative to the two last cited works, the proposed frequency sweeping algorithm enables to seek a better performance ensuring that the control efforts remain between the physical limits, the reader may observe the current and voltages responses.

\section{CONCLUSION}

This paper deals with the study of the two robust control approaches based in $\mathcal{H}_{\infty}$ norm and applied to improve the performance and stability of an induction motor. The first

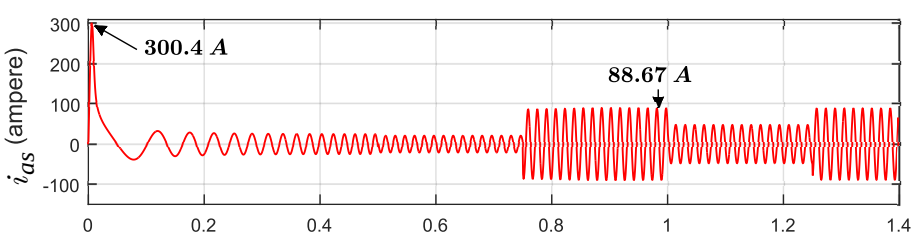

(a)
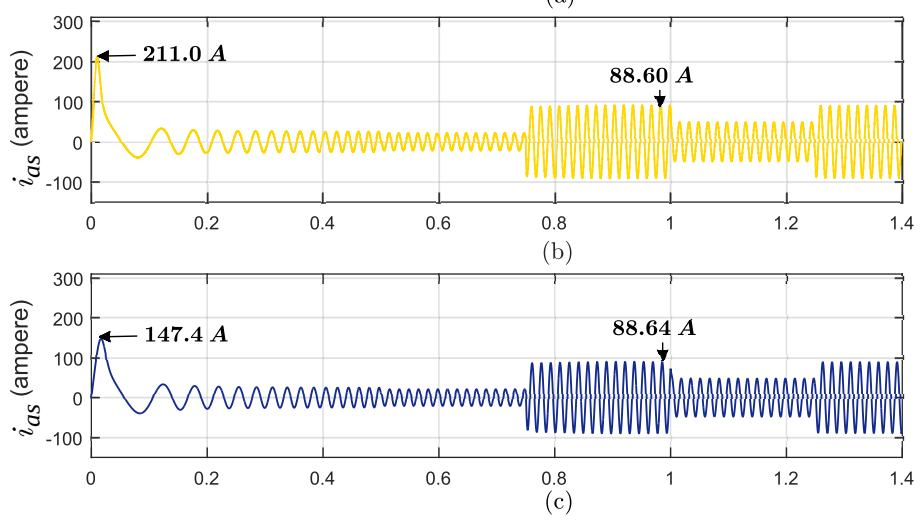

Time (s)

Fig. 8. The Stator Current $i_{a s}$ Responses with $\mathcal{H}_{\infty}$ Controllers: (a) Structured Controller, (b) Unstructured Controller, (c) PI Conventional Controller.

approach, the unstructured $\mathcal{H}_{\infty}$ control presents quite good responses, included robustness properties. The second approach, the structured $\mathcal{H}_{\infty}$ control approach presents better responses relative to the conventional PI controller. However, its constraints in its defined structure limits a suboptimal solution regarding robustness and may compromise the disturbance rejection. The proposed sweep frequency algorithm, used in the two approaches, seeks a gamma suboptimal value close to 1 , iterating the solution relative to the natural frequency. The structured controller is more attractive for practitioners due to its straightforward implementation; but its robustness properties should be studied in a further work considering more parameters and using a testing bench. Results indicate the good election for robustness design is still the unstructured controller.

\section{ACKNOWLEDGMENT}

The authors thank the Universidad Nacional de San Agustín de Arequipa for supporting this research under incentives for research publication.

\section{REFERENCES}

[1] M. Aktas, K. Awaili, M. Ehsani, and A. Arisoy, "Direct torque control versus indirect field-oriented control of induction motors for electric vehicle applications," Engineering Science and Technology, an International Journal, vol. 23, no. 5, pp. 1134-1143, 2020. [Online]. Available: https://www.sciencedirect.com/science/article/pii/S2215098619326734

[2] H. Guessoum, C.-E. Feraga, L. Mehennaoui, M. Sedraoui, and A. Lachouri, "A robust performance enhancement of primary $\mathrm{h} \infty$ controller based on auto-selection of adjustable fractional weights: Application on a permanent magnet synchronous motor," Transactions of the Institute of Measurement and Control, vol. 41, no. 11, pp. 3248-3263, 2019. [Online]. Available: https://doi.org/10.1177/0142331218823861 
[3] A. Devanshu, M. Singh, and N. Kumar, "Sliding mode control of induction motor drive based on feedback linearization," IETE Journal of Research, vol. 66, no. 2, pp. 256-269, 2020. [Online]. Available: https://doi.org/10.1080/03772063.2018.1486743

[4] D. C. Happyanto, A. Aditya, and B. Sumantri, "Boundary-layer effect in robust sliding mode control for indirect field oriented control of 3phase induction motor," International Journal on Electrical Engineering and Informatics, vol. 12, pp. 188-204, 2020.

[5] C. Paterson, I. Postlethwaite, D. Walker, and A. Bashagha, "The development and evaluation of an h-infinity induction motor controller," IFAC Proceedings Volumes, vol. 29, no. 1, pp. 3368-3373, 1996, 13th World Congress of IFAC, 1996, San Francisco USA, 30 June - 5 July. [Online]. Available: https://www.sciencedirect.com/science/article/pii/S1474667017581975

[6] E. Prempain and I. Postlethwaite, " $\mathrm{H} \infty$, design for an induction motor," IFAC Proceedings Volumes, vol. 35, no. 1, pp. 211216, 2002, 15th IFAC World Congress. [Online]. Available: https://www.sciencedirect.com/science/article/pii/S1474667015387905

[7] C.-E. Feraga, M. Sedraoui, and R. Bachir Bouiadjra, "Enhanced indirect field-oriented control of single-phase induction motor drive using h $\infty$ current controller," Arabian Journal for Science and Engineering, vol. 44, no. 8, pp. 7187-7202, 2019. [Online]. Available: https://doi.org/10.1007/s13369-019-03850-6

[8] J. K. Jain, S. Ghosh, S. Maity, and P. Dworak, "Pi controller design for indirect vector controlled induction motor: A decoupling approach," ISA Transactions, vol. 70, pp. 378-388, 2017. [Online]. Available: https://www.sciencedirect.com/science/article/pii/S001905781730438X

[9] J. Jain, S. Ghosh, and S. Maity, "Concurrent pi controller design for indirect vector controlled induction motor: Concurrent pi controller design," Asian Journal of Control, vol. 22, 092018.

[10] C. M. Ionescu, E. H. Dulf, M. Ghita, and C. I. Muresan, "Robust controller design: Recent emerging concepts for control of mechatronic systems," Journal of the Franklin Institute, vol. 357, no. 12, pp. 7818-7844, 2020. [Online]. Available: https://www.sciencedirect.com/science/article/pii/S0016003220303999

[11] A. A. Z. Diab, A.-H. M. El-Sayed, H. H. Abbas, and M. A. E Sattar, "Robust speed controller design using $h$ infinity theory for high-performance sensorless induction motor drives," Energies, vol. 12, no. 5, 2019. [Online]. Available: https://www.mdpi.com/1996$1073 / 12 / 5 / 961$

[12] G. Rigatos, P. Siano, and M. Abbaszadeh, "Nonlinear optimal control for ship propulsion systems comprising an induction motor and a drivetrain," Proceedings of the Institution of Mechanical Engineers, Part M: Journal of Engineering for the Maritime Environment, vol. 234, no. 2, pp. 409-425, 2020. [Online]. Available: https://doi.org/10.1177/1475090219885213

[13] E. Zhao, J. Yu, J. Liu, and Y. Ma, "Neuroadaptive dynamic surface control for induction motors stochastic system based on reduced-order observer," ISA Transactions, 2021. [Online]. Available: https://www.sciencedirect.com/science/article/pii/S0019057821004900

[14] M. A. Mossa and H. Echeikh, "A novel fault tolerant control approach based on backstepping controller for a five phase induction motor drive: Experimental investigation," ISA Transactions, vol. 112, pp. 373-385, 2021. [Online]. Available: https://www.sciencedirect.com/science/article/pii/S0019057820305188

[15] P. Alkorta, J. A. Cortajarena, O. Barambones, and F. J. Maseda, "Effective generalized predictive control of induction motor," ISA Transactions, vol. 103, pp. 295-305, 2020. [Online]. Available: https://www.sciencedirect.com/science/article/pii/S0019057820301610

[16] Z. Yang, Q. Ding, X. Sun, H. Zhu, and C. Lu, "Fractionalorder sliding mode control for a bearingless induction motor based on improved load torque observer," Journal of the Franklin Institute, vol. 358 , no. 7 , pp. 3701-3725, 2021. [Online]. Available: https://www.sciencedirect.com/science/article/pii/S0016003221001733

[17] C.-M. Ong et al., Dynamic simulation of electric machinery: using MATLAB/SIMULINK. Prentice hall PTR Upper Saddle River, NJ, 1998, vol. 5 .

[18] S. Skogestad and I. Postlethwaite, Multivariable feedback control: analysis and design. Citeseer, 2007, vol. 2.

[19] P. Apkarian and D. Noll, "Nonsmooth h $\infty$ synthesis," IEEE Transactions on Automatic Control, vol. 51, no. 1, pp. 71-86, 2006.

[20] P. Gahinet and P. Apkarian, "Decentralized and fixed-structure $\mathrm{h} \infty$ control in matlab," in 2011 50th IEEE Conference on Decision and Control and European Control Conference, 2011, pp. 8205-8210. 


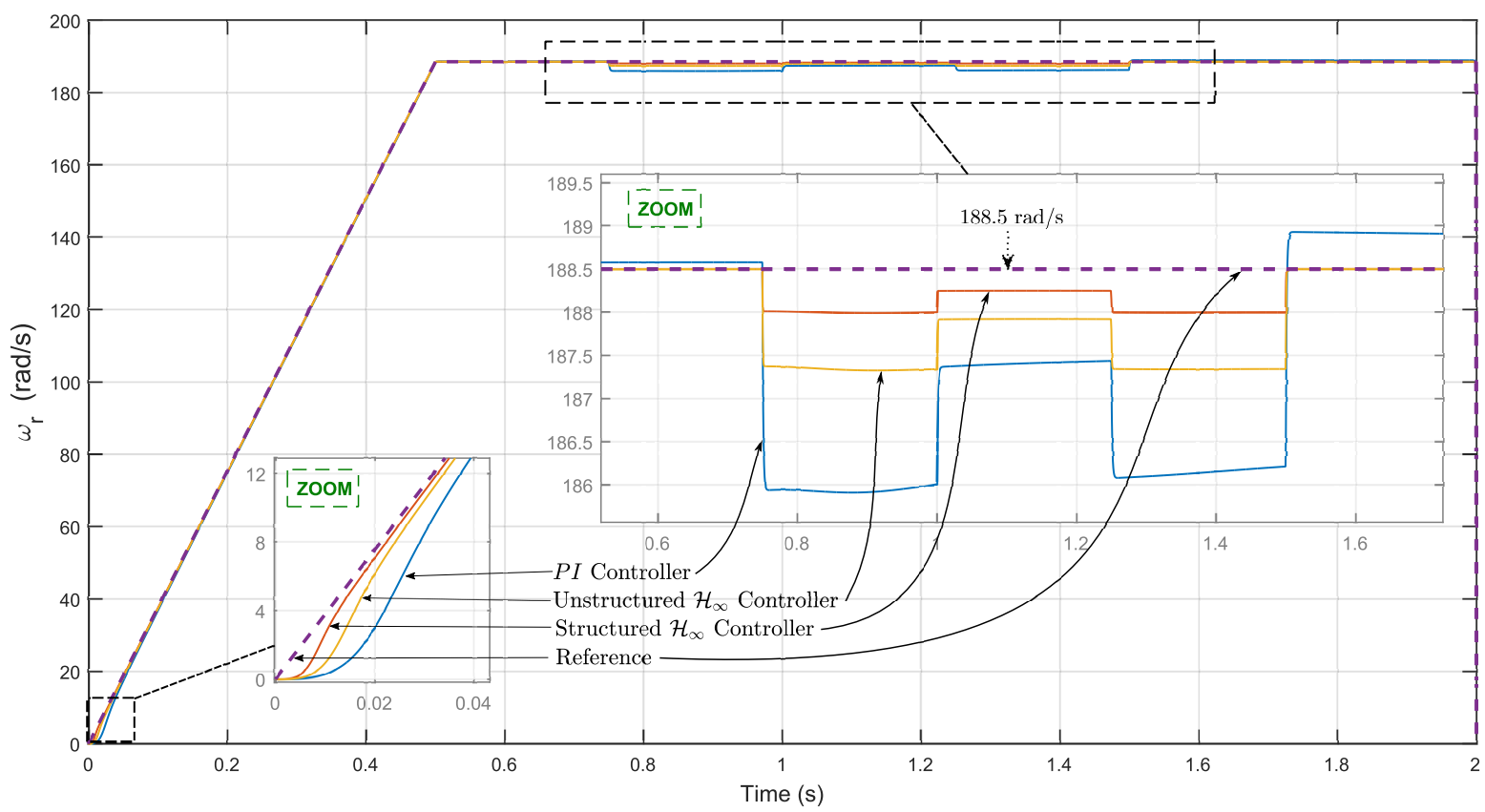

Fig. 9. Controlled Variable Comparison, Rotor Speed $\omega_{r}$.

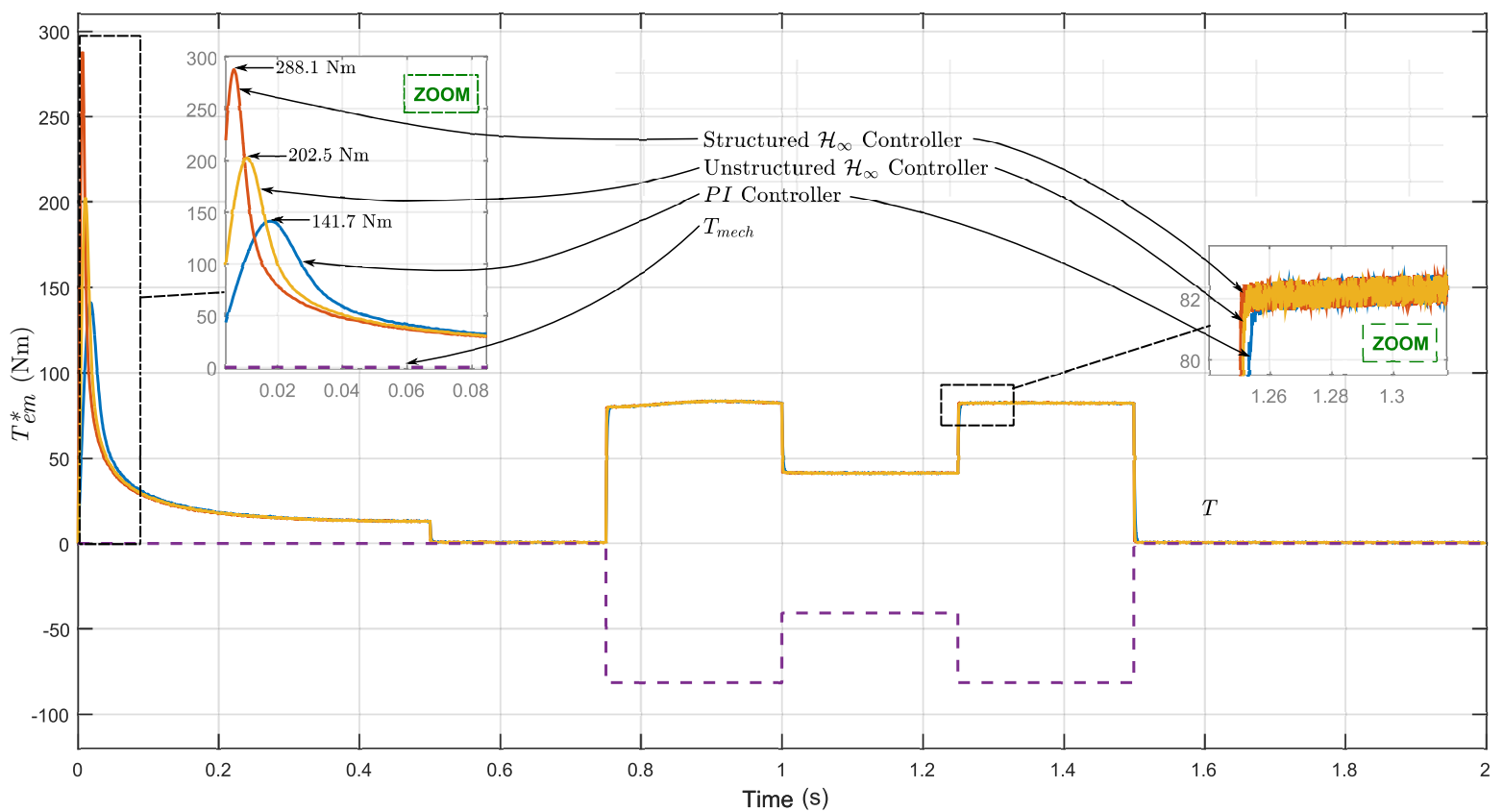

Fig. 10. Control Variable Comparison, Electromagnetic Torque $T_{e m}^{*}$ Compensates the Required Mechanical Torque $T_{m e c h}$. 